\title{
Direction of Arrival Estimation Based on MUSIC Algorithm Using Uniform and Non-Uniform Linear Arrays
}

\author{
Eva Kwizera $^{1}$, Elijah Mwangi ${ }^{2}$ and Dominic B.O. Konditi ${ }^{3}$ \\ ${ }^{1}$ Department of Electrical Engineering, Pan African University, Kenya, P.O. Box 62000-002000, Nairobi-Kenya \\ ${ }^{2}$ School of Engineering, University of Nairobi, P.O. Box 30197-00100, Nairobi-Kenya \\ ${ }^{3}$ Department of Telecommunication and Information Engineering, Technical University of Kenya, P.O. Box \\ 52428-00200, Nairobi-Kenya
}

\begin{abstract}
In signal processing, the direction of arrival (DOA) estimation denotes the direction from which a propagating wave arrives at a point, where a set of antennas is located. Using the array antenna has an advantage over the single antenna in achieving an improved performance by applying Multiple Signal Classification (MUSIC) algorithm. This paper focuses on estimating the DOA using uniform linear array (ULA) and non-uniform linear array (NLA)of antennas to analyze the performance factors that affect the accuracy and resolution of the system based on MUSIC algorithm. The direction of arrival estimation is simulated on a MATLAB platform with a set of input parameters such as array elements, signal to noise ratio, number of snapshots and number of signal sources. An extensive simulation has been conducted and the results show that the NLA with DOA estimation for co-prime array can achieve an accurate and efficient DOA estimation.

Keywords: Direction of Arrival (DOA) estimation, Multiple Signal Classification (MUSIC), Root-MUSIC, Non-Uniform Linear Array (NLA), Uniform Linear Array (ULA).
\end{abstract}

\section{INTRODUCTION}

In the past decades, many array models such as the uniform linear array (ULA), uniform circular array, uniform rectangular array, and nonuniform linear array (NLA) have been utilized towards the achievement of the direction of arrival (DOA) estimation[1]. To get an accurate DOA estimation of the narrowband signal sent from a far field source to a receiving array antenna can increase the wireless communication systems capacity [2]. Therefore, putting effort on improving DOA estimation methods isa key to developing the quality of wireless networks.

Many methods have been employedto solve such problems among them the maximum likelihood (ML) method of Capon (1969) and Burg's maximum entropy (ME) method which are among the non-subspace techniques [3], [4]. Although they have been often successful and widely used, these methods have certain fundamental limitations especially the bias and sensitivity in parameter estimates, largely because they use an incorrect model of the measurements [5]. Then, Schmidt in1979[6]corrected the measurement model in the case of sensor arrays of arbitrary form and proposes the subspace technique termed Multiple Signal Classification (MUSIC) algorithm. It is based on exploiting the eigen structure of the input covariance matrix. The DOA of multiple source signals can be easily estimated by identifying the peaks of a MUSIC spatial spectrum. Thereafter, for reduction of computational complexity of MUSIC, RootMUSIC algorithm was developed by Bara bell in 1983[7]. It is based on polynomial rooting giving higher resolution, but it is possible to use it when only a uniform linear array is present. For the small number of array elements with also small value of signal to noise ratio, the Root- MUSIC is more accurate compared to MUSIC.

Recently, a non-uniform linear array in a form of co-prime array has been proposed[8]. Its most remarkable property is that it increases the degrees of freedom. In addition, the autocorrelation of signals can be estimated in a much denser spacing other than the physically sparse sampling spacing, and sinusoids in noise can be estimated in a more effective way. Due to the useful properties of the NLA, its importance has been realized and has been the object of research in the last few years. Some researchers such as Paland Vaidyanathan in [9], [10] proposed a new method for a super resolution spectral estimation from the perspective of degree of freedom increase. Further to these efforts, Wengand Petar in [11]proposed a search-free DOA algorithm for co-prime arrays by using a projection-like method to eliminate the phase ambiguities for obtaining the unique estimation of DOA.

In this paper, a method of estimation of DOA by comparing the performance analysis using uniform and non-uniform linear array antennas is proposed. It underlies the factors that affect the accuracy and resolution of the system based on 
multiple signal classification (MUSIC) and rootMUSIC algorithms. The performance evaluation of the MUSIC algorithm under ULA and NLA is given.

The remainder of this paper is organized as follows. In Section 2, the signal model of the uniform linear array is presented. In Section 3, the description of a detailed MUSIC algorithm implementation is given. Section 4, shows a nonuniform linear array with combined MUSIC algorithm. Simulation and discussion of the results are presented in Section 5. Finally, conclusion and recommendation for future work is highlighted in Section 6.

\section{SIGNAL MODEL OF UNIFORM LINEAR ARRAY}

Consider a linear array antenna with $M$ antenna elements that are equally spaced with a distanced that is strictly equal to a half wavelength $\left(d=\frac{\pi}{2}\right)$.Assume that there are narrowband signal sources $(K)$ located at $\theta_{1}, \theta_{2}, \ldots, \theta_{K}$ with signal powers $\sigma_{1}^{2}, \sigma_{2}^{2}, \ldots, \sigma_{K}^{2}$. The incident signals and the noise are uncorrelated. In addition the incident signals are themselves uncorrelated. Let the number of signal sources be less than the number of antenna elements $(K<M)$.

The steering vector for $k^{t \square}$ source located at $\theta_{k}$ is

with

$$
a\left(\theta_{k}\right) \Theta \square^{M \times 1} \text { with } \quad k^{\text {tロ }} \text { element }
$$

$e^{j(2 \pi / \lambda) d_{1} \sin \left(\theta_{k}\right)}$, where $d_{l}$ is the antennas location and $\lambda$ is the wavelength as shown in the fig. 1 .

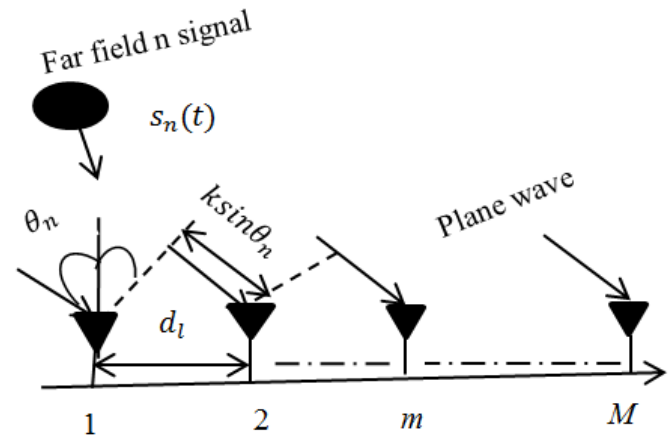

Fig.1. A plane wave incident on a uniform linear array antenna
The signal collected by all antennas at time can be expressed as

$X(t)=A s(t)+n(t)$

$$
\text { Where } X(t)=\left[x_{1}(t) x_{2}(t) \ldots x_{M}(t)\right]^{T} \text { is a }
$$
vector received by the array antenna,

$A=\left[a\left(\theta_{1}\right) a\left(\theta_{2}\right) \ldots a\left(\theta_{K}\right)\right]$ is a steering vector. Its expression is shown in $(2), s(t)$ is the signal vector generated by the source, $n(t)=\left[n_{1}(t) n_{2}(t) \ldots \ldots n_{M}(t)\right]^{T}$ and is the additive white Gaussian noise. $a\left(\theta_{k}\right)=$

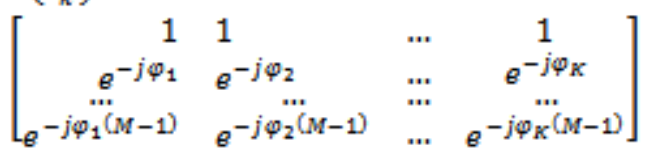

(2)

$w$ पere $\varphi_{k}=\frac{2 \pi d}{\lambda} \sin \theta_{k}$

The narrowband signal from (1) can be expressed as:

$S_{k}(t)=s_{k}(t) \exp \left\{j \omega_{k}(t)\right\}$.

Where $s_{k}(t)$ is the complex envelope of $S_{k}(t)$ and $\omega_{k}(t)$ is the angular frequency of $S_{k}(t)$.

As assumed all signals have the same center frequency, therefore:

$\omega_{k}=\omega_{0}=\frac{2 \pi c}{\lambda}$

Where $c$ is the speed of the light and $\lambda$ is the wavelength of the signal.

According to the narrowband assumption, the following approximation is valid:

$s_{k}\left(t-t_{1}\right) \approx s_{k}(t)$

The delayed wavefront signal is:

$s_{k}\left(t-t_{1}\right)=s_{k}(t) \exp \left[j \omega_{0}\left(t-t_{1}\right)\right]=s_{k}(t) \exp \left[j \omega_{0}\left(t-t_{1}\right)\right]$

The output signal of the $m^{\text {th }}$ element is:

$X_{m}(t)=$

$=\sum_{k=1}^{K} s_{k}(t) \exp \left[-j(m-1) \frac{2 \pi d \sin \theta_{k}}{\lambda}\right]+n_{m}(t)$

From (6) the output steering array vector is:

$a_{m}(t)=\exp \left[-j(m-1) \frac{2 \pi d \sin \theta_{k}}{\lambda}\right]$

\section{MUSIC ALGORITHM IMPLEMENTATION}

Multiple signal classification is a subspace technique based on exploiting the eigenstructure of input covariance matrix [12]. Through a set of input parameters such as number of array elements, number of snapshots, element spacing, angular 
separation, signal-to-noise ratio and MUSIC algorithm, the DOA is estimated with high resolution. This leads to high quality of wireless communication.

The autocorrelation of the received signal is:

$R_{x}=E\left[X X^{H}\right]$

Where $H$ is the conjugate transpose matrix. The noise is assumed to be zero mean and additive white Gaussian and is uncorrelated to the signal.

Replacing (1) into(9), the covariance matrix is obtained:

$$
\begin{aligned}
& R_{x}=E\left[(A s+N)(A s+N)^{H}\right] \\
& =A E\left[s s^{H}\right] A^{H}+E\left[N N^{H}\right] \\
& =A R_{S} A^{H}+R_{N}
\end{aligned}
$$

Where $R_{s}=E\left[s s^{H}\right]$ is called source signal correlation matrix, $R_{N}=\sigma^{2} I$ is a noise correlation matrix.

If $\left(\lambda_{1}, \lambda_{2}, \ldots . \lambda_{M}\right)$ are eigenvalues of spatial correlation matrix $R_{x}$; Then the performance of eigenvalue associated with a particular eigenvector is given as:

$$
R_{x}-\lambda_{\mathrm{i}} \mathrm{I}=0
$$

$A R_{S} A^{H}+\sigma^{2} I-\lambda_{i} I=0$

$A R_{S} A^{H}+\left(\sigma^{2}-\lambda_{i}\right) \mathrm{I}=0$

Therefore, eigenvectors $\nu_{\mathrm{i}}$ of $A R_{S} A^{H}$ are obtained using (14);

$v_{i}=\sigma^{2}-\lambda_{i}$

The eigenvalues are sorted accordingto their magnitudes $\lambda_{1} \geq \lambda_{2} \geq \cdots \geq \lambda_{M} \geq 0$. The larger eigenvalues correspond to signal subspace, while small eigenvalues corresponds to noise. The largest eigenvalues are all values greater than $K$ that is $(M-K)$, and the rest are all valuesthat are less than $K$.Thus, MUSIC "Spatial spectrum" in [12] is defined as:

$$
P_{\text {music }}(\theta)=\frac{1}{a^{H}(\theta) E_{N} E_{N}{ }^{H} a(\theta)}
$$

\section{NON-UNIFORM LINEAR ARRAY WITH COMBINED MUSIC \\ ALGORITHM}

A system with the array antennas linearly spaced,the different array elements spacing and the signal phase differencesis considered. In this case,one non-uniform linear arraycan be decomposed into two uniform linear arrays as shown in fig. 2 and fig. 3 respectively. The direction of arrival is estimated by combining MUSIC results from the two co-prime arrays.

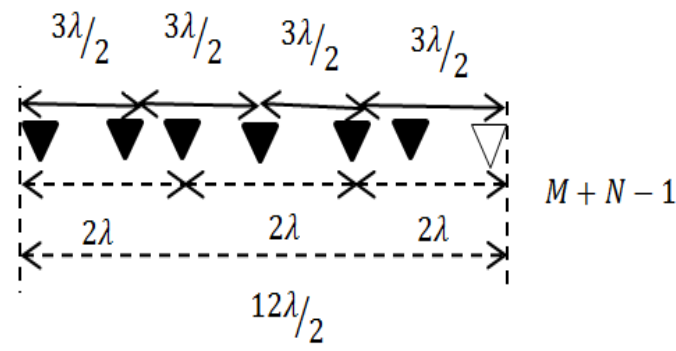

Fig. 2.Co-prime non-uniform linear array

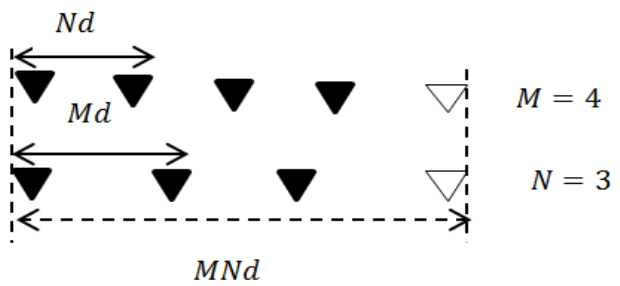

Fig.3.Two co-prime uniform linear arrays

The received signal vector of each sub-array at the $t$-th time slot can be defined as:

$X_{M}(t)=A_{M} s(t)+n(t)$

$X_{N}(t)=A_{N} s(t)+n(t)$

The array output vector of the co-prime array is given by:

$Y(t)=A(t) s(t)+n(t)$.

According to the far-field assumption, the steering vector corresponding to the $k^{\text {th }}$ source is:

$a_{k}$

$=\left[1, e^{-j \frac{2 \pi}{\lambda} d_{1} \sin \left(\theta_{k}\right)}, \ldots, e^{-j \frac{2 \pi}{\lambda} d_{i} \sin \left(\theta_{k}\right)}, e^{-j \frac{2 \pi}{\lambda} d_{(M+N-1)} \sin \left(\theta_{k}\right)}\right]^{Y}$

(19)

Whered $d_{i}(l=1,2, \ldots, M+N-1)$

Steering arrays of each linear array are given by:

$a_{M k}=$

$=\left[1_{s} e^{-j \pi N \sin \left(\theta_{k}\right)}{ }_{{ }_{n} \ldots,} e^{-j \pi(M-1) N \sin \left(\theta_{k}\right)}\right]^{T}$

$a_{\mathrm{Nk}}=$

$=\left[1_{s} e^{-j \pi M \sin \left(\theta_{k}\right)}{ }_{s, n} e^{-j \pi(N-1) M \sin \left(\theta_{k}\right)}\right]^{T}$

By obtaining each sample of covariance matrices from(16) and (17), the two decomposed uniform linear sub-arrays give:

$R_{x M}=E\left[X_{M}(t) X_{M}(t)^{H}\right]$
$R_{x N}=E\left[X_{N}(t) X_{N}(t)^{H}\right]$

Replacing the received signal vector of each subarray(16) and (17) by their values,the following is obtained: 
$R_{x M}=E\left[\left(A_{M} s(t)+n(t)\right)\left(A_{M} s(t)+n(t)\right)^{H}\right]$

$=A_{M} E\left(s(t) s(t)^{H}\right) A_{M}{ }^{H}+E\left[N N^{H}\right]$

$=A_{M} R_{S M} A_{M}{ }^{H}+R_{N}$

$R_{x N}=E\left[\left(A_{N} s(t)+n(t)\right)\left(A_{N} s(t)+n(t)\right)^{H}\right]$

$=A_{N} E\left(s(t) s(t)^{H}\right) A_{N}{ }^{H}+E\left[N N^{H}\right]$

$=A_{N} R_{S N} A_{N}{ }^{H}+R_{N}$

Where $R_{S M}=E\left[s(t) s(t)^{H}\right]$ and

$R_{S N}=E\left[s(t) s(t)^{H}\right]$ are source signal correlation matrices of subarrays $M$ and $N$ respectively, $R_{\mathbb{N}}=\sigma^{2} I$ is a noise correlation matrix for each subarray.

If $\left(\lambda_{1}, \lambda_{2}, \ldots . \lambda_{M}\right)$ and $\left(\lambda_{1}, \lambda_{2}, \ldots . \lambda_{N}\right)$ are eigenvalues of the spatial correlation matrix $R_{x M}$ and $R_{x N}$, then the performance of eigenvalue associated with a particular eigenvector for each sub-array is given as:

$R_{x}-\lambda_{\mathrm{i}} \mathrm{I}=0$

$A_{M} R_{S M} A_{M}{ }^{H}+\left(\sigma^{2}-\lambda_{i}\right) I=0$

$A_{N} R_{S N} A_{N}{ }^{H}+\left(\sigma^{2}-\lambda_{i}\right) \mathrm{I}=0$

The eigenvectors $v_{i}$ of $A_{M} R_{S M} A_{M}{ }^{H}$ and $A_{N} R_{S N} A_{N}{ }^{H}$ from (24) and (25) are obtained using (36) whereby $v_{\mathrm{i}}$ is:

$v_{i}=\sigma^{2}-\lambda_{i}$

Therefore applying eigen-decomposition to the sample covariance matrices in (24) and (25) yields:

$R_{x M}=E_{g M} \Lambda_{g M} E_{g M}{ }^{H}+E_{n M} \Lambda_{n M} E_{n M}{ }^{H}$

$R_{x N}=E_{g N} A_{g N} E_{g N}{ }^{H}+E_{n N} A_{n N} E_{n N}{ }^{H}$

Then, according to the orthogonality between the signalsubspace and the noise subspace of each subarray, the MUSIC spatial pseudo-spectrum of the two decomposed linear subarrays $M$ and $N$ respectively, are:

$$
\begin{aligned}
& P_{M U S I C}(\theta)=\frac{1}{a_{M}(\theta)^{H} E_{n M} E_{n M}{ }^{H} a_{M}(\theta)} \\
& P_{M U S I C_{N}}(\theta)=\frac{1}{a_{N}(\theta)^{H} E_{n N} E_{n N}{ }^{H} a_{N}(\theta)}
\end{aligned}
$$

\section{SIMULATION RESULTS AND DISCUSSION}

In this session, computer simulations are provided to substantiate the performance analysis of uniform and non-uniform linear arrays. In all cases, the impinging source signals are narrowband signals operating at the same center frequency $2.4 \mathrm{GHz}$ the similar to the one used in wireless communication (wi-fi), with the azimuth arrival angle interval in the range: $[-\pi / 2, \pi / 2]$. These signals are uncorrelated with themselves and with the noise as well. The additive background noise is assumed to be spatially and temporarily uncorrelated white complex Gaussian with the zero-mean.

These simulations are subdivided into three parts. Firstly the validation of the results using existing data in the literature was given, then the variations of the direction of arrival with number of snapshots and with number of array elements, for both ULA and NLA are simulated. This is to study the performance evaluation and the accuracy of the direction of arrival estimation for each array arrangement.

\section{a. Validation of results}

The simulation results were justified by using existing data in the literature.The dashed lines in fig. 4 are results represented by Dhering[7] and the continuous lines are the present work with the increased signal to noise ratio. As observed, there is a good agreement between the simulated results and the published data by Dhering.

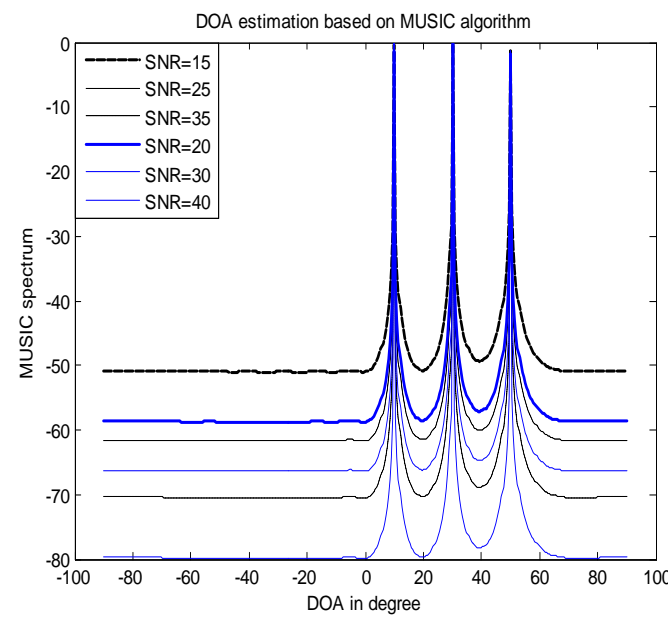

Fig. 4: variation of DOA estimation with SNR

\section{b. Experiment 1: Variation of DOA with the snapshots}

In experiment 1 , the variation of the DOA estimation with the number of snapshots is simulated. For uniform linear array, the number of snapshots was varied from $\mathrm{N}=100$ to $\mathrm{N}=1000$ snapshots in steps of 100 snapshots. The results of the DOA for $\mathrm{N}=100,500$ and 1000 snapshots are given in fig 5. This simulation is based on MUSIC algorithm. During the simulation of this experiment, the following signal and array parameters are kept constant:

True DOA: $60^{\circ}$ and $68^{\circ}$, signal frequency: Freq $=2.4 \mathrm{GHz}, \mathrm{SNR}=-5 \mathrm{~dB}$, Array size: $\mathrm{M}=11$. 


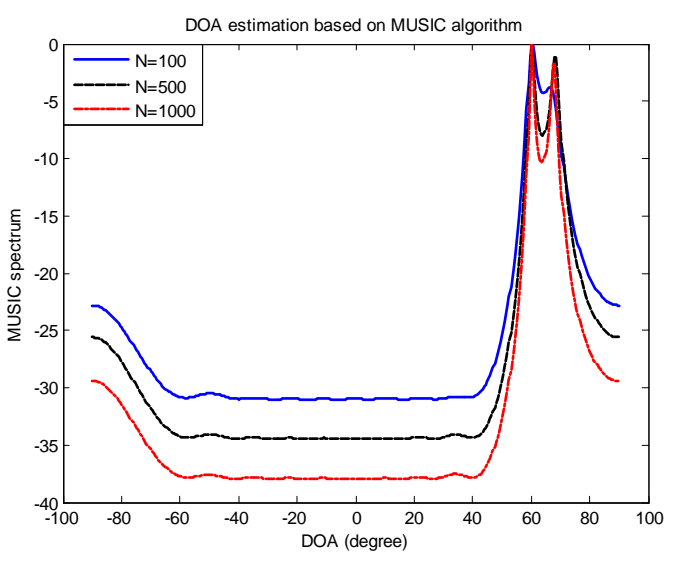

Fig. 5: Variation of DOA estimation with snapshots

The results from fig 5 can be summarized in Table 1. It can be noted that with $N=100$ snapshots, the estimated DOA is $60.5^{\circ}$ and $67^{\circ}$ respectively; Also, with $N=500$ snapshots the estimated DOA is $59.5^{\circ}$ and $68^{\circ}$ respectively; with $N=1000$ snapshots the estimated DOA is $60^{\circ}$ and $68.5^{\circ}$ respectively.

Table 1: Estimated DOA with snapshots for ULA

\begin{tabular}{|l|l|l|}
\hline No of snapshots & $\hat{\theta}_{1}$ (degree) & $\hat{\theta}_{2}$ (degree) \\
\hline 100 & 60.5 & 67 \\
\hline 500 & 59.5 & 68 \\
\hline 1000 & 60 & 69 \\
\hline
\end{tabular}

The simulation for the non - uniform linear array with DECOM $(7,5)$, the number of snapshots was varied from $N=100$ to $N=1000$ snapshots in steps of 100 snapshots. The results of the DOA for $N=100,500$ and 1000 snapshots are given in fig. 6 , fig. 7 and fig. 8respectively.

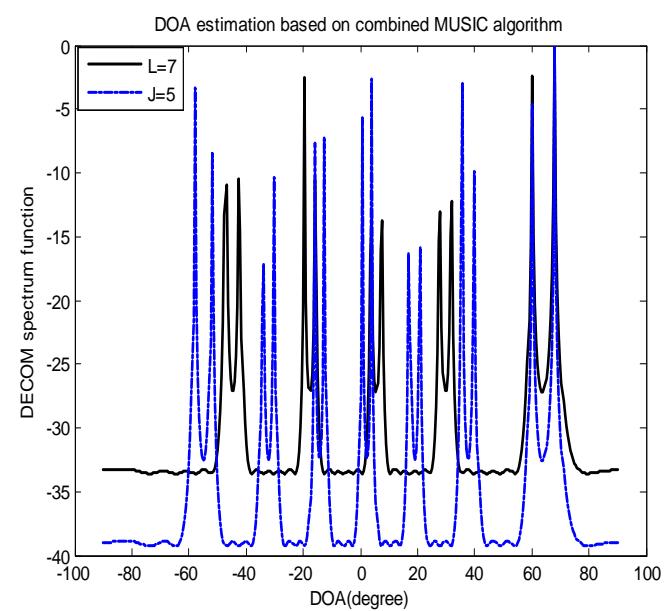

Fig. 6: DOA estimation with 100 snapshots using $\operatorname{DECOM}(7,5)$

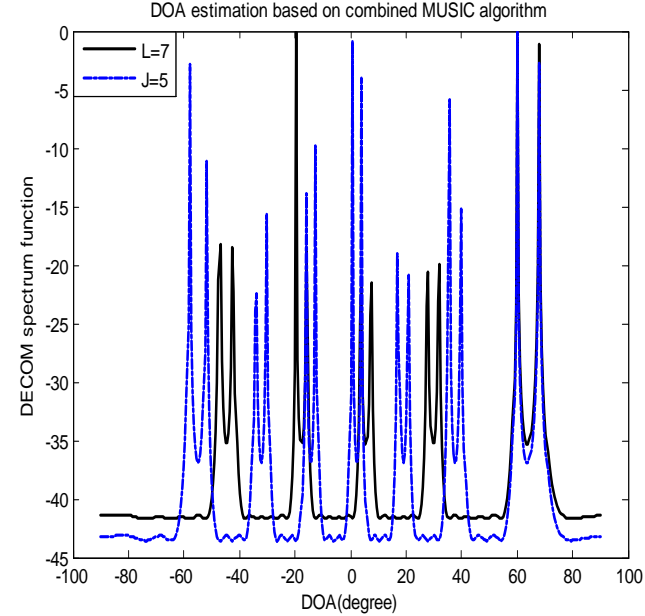

Fig. 7: DOA estimation with 500 snapshots using $\operatorname{DECOM}(7,5)$

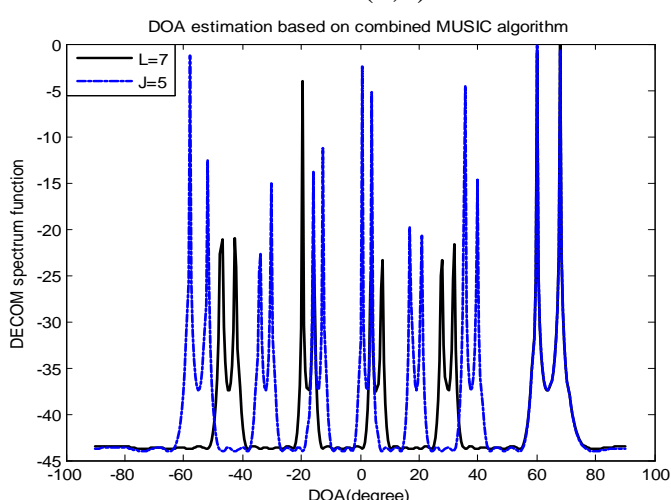

Fig.8: DOA estimation with 1000 snapshots using $\operatorname{DECOM}(7,5)$

The results from fig. 6 , fig. 7 and fig. 8 can be summarized in Table 2. It can be noted that with $N=100$ snapshots, the estimated DOA is $60^{\circ}$ and $68^{\circ}$ respectively; Also, with $N=500$ snapshots the estimated DOA is $60^{\circ}$ and $68^{\circ}$ respectively; with $N=1000$ snapshots the estimated DOA is $60^{\circ}$ and $68^{\circ}$ respectively.

Table 2: Estimated DOA with snapshots for DECOM

\begin{tabular}{|l|l|l|}
\hline No of snapshots & $\widehat{\theta}_{1}$ (degree) & $\widehat{\theta}_{2}$ (degree) \\
\hline 100 & 60 & 68 \\
\hline 500 & 60 & 68 \\
\hline 1000 & 60 & 68 \\
\hline
\end{tabular}

\section{c. Experiment 2: Variation of DOA with array elements}

In experiment 2 , the variation of the DOA estimation with the number of array size is simulated. For uniform linear array, the number of array element was varied from $M=3$ elements to $M=11$ elements in steps of 2 elements. The results of the DOA for $M=3,6$ and 11 elements are given in fig 4.10. This simulation is based on 
MUSIC algorithm. During the simulation of this experiment, the following signal and array parameters are kept constant:

TrueDOA: $60^{\circ}$ and $68^{\circ}$, signal frequency: Freq $=$ $2.4 \mathrm{GHz}$, snapshots: $\mathrm{N}=100$, SNR $=-5 \mathrm{~dB}$

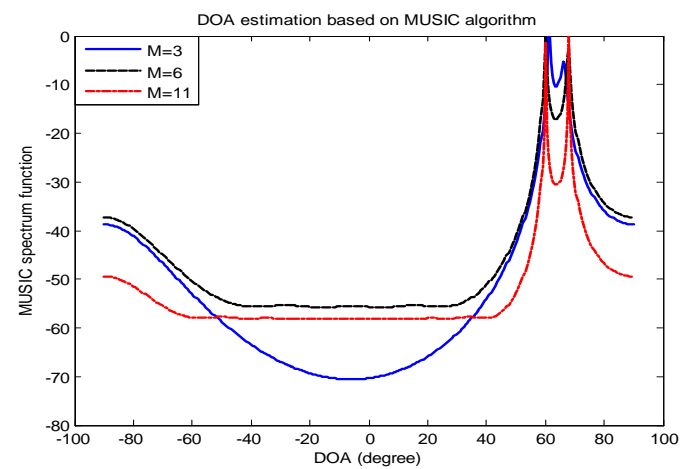

Fig. 9: DOA estimation with array elements variation

The results from fig. 9 can be summarized in Table 5. It can be noted that with $M=3$ elements, the estimated DOA is $61^{\circ}$ and $66^{\circ}$ respectively; Also, with $M=6$ elements the estimated DOA is $60^{\circ}$ and $68.5^{\circ}$ respectively; with $M=11$ the estimated DOA is $60^{\circ}$ and $68^{\circ}$ respectively.

Table 3: Estimated DOA with the array elements for ULA

\begin{tabular}{|l|l|l|}
\hline$M$ & $\hat{\theta}_{1}$ (degree) & $\widehat{\theta}_{2}$ (degree) \\
\hline 3 & 61 & 66 \\
\hline 6 & 60 & 68.5 \\
\hline 11 & 60 & 68 \\
\hline
\end{tabular}

The simulation for the non -uniform linear array, the array size was varied. The results of the DOA for $\operatorname{DECOM}(4,3), \operatorname{DECOM}(9,4), D E C O M$ $(11,5)$ are given are in fig. 10 , fig. 11 , fig. 12 respectively.

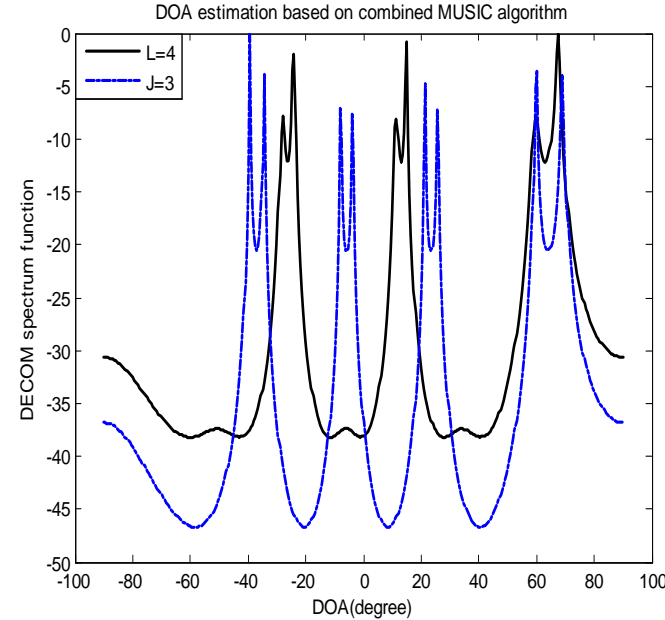

Fig. 10: Variation of DOA estimation with $\operatorname{DECOM}(4,3)$

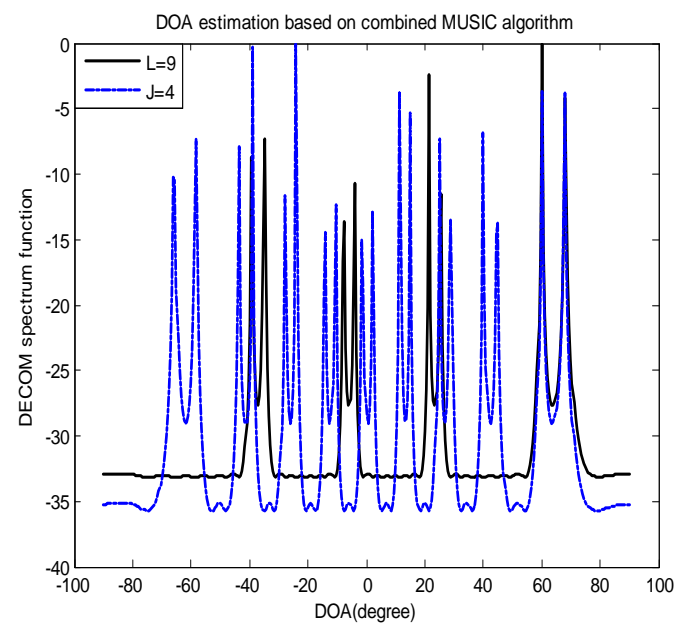

Fig. 11: Variation of DOA estimation with $\operatorname{DECOM}(9,4)$

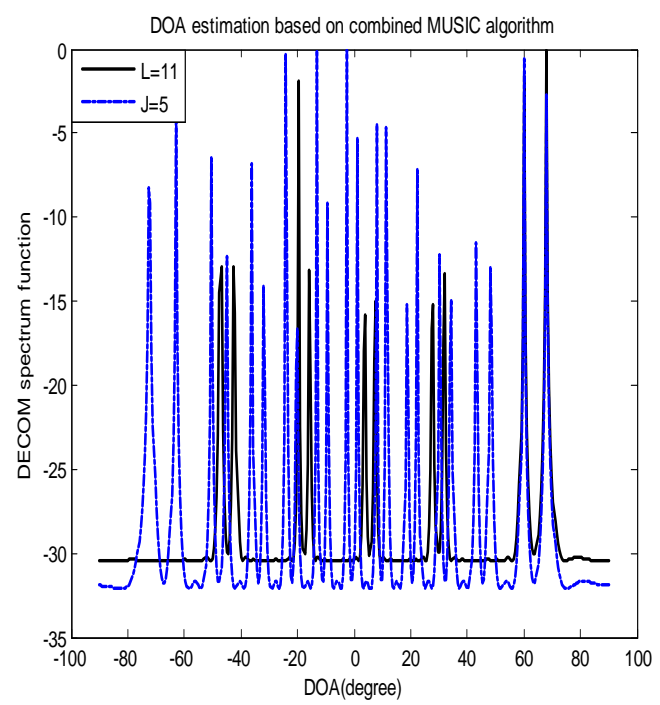

Fig. 12: Variation of DOA estimation with $\operatorname{DECOM}(11,5)$ 
The results from fig. 10, fig. 11, fig. 12 can be summarized in Table 6 . It can be noted that with $\operatorname{DECOM}(4,3)$, the estimated DOA for 4 elements is $59.5^{\circ}$ and $67.5^{\circ}$, for 3 elements is $60^{\circ}$ and $69^{\circ}$ respectively; Also, with $\operatorname{DECOM}(9,4)$ the estimated DOA for 9 elements is $60^{\circ}$ and $68^{\circ}$, for 4 elements is $60^{\circ}$ and $68.5^{\circ}$ respectively; with $\operatorname{DECOM}(11,5)$ the estimated DOA for 11 elements is $60^{\circ}$ and $68^{\circ}$, for 5 elements is $60^{\circ}$ and $68^{\circ}$ respectively.

Table 4: Estimated DOA with signal to noise ratio for DECOM

\begin{tabular}{|l|l|l|l|l|}
\hline $\operatorname{DECOM}$ & $\hat{\theta}_{1}$ & $\hat{\theta}_{2}$ & $\hat{\theta}_{1}$ & $\hat{\theta}_{1}{ }^{{ }^{\prime}}$ \\
\hline $\operatorname{DECOM}(4,3)$ & 59.5 & 67.5 & 60 & 69 \\
\hline $\operatorname{DECOM}(9,4)$ & 60 & 68 & 60 & 68 \\
\hline $\operatorname{DECOM}\left(11_{s}, 5\right)$ & 60 & 68 & 60 & 68 \\
\hline
\end{tabular}

\section{d. Discussion}

A comparative study has been made between uniform linear array and non-uniform linear array. The computer simulation are depicted to show the performance evaluation of MUSIC and Root-MUSIC algorithms based on variation of DOA estimation with number of snapshots, signal to noise ratio, number of array elements and number of signal sources. The simulation results were divided into five experiments.

The experiment 1 shows the variation of DOA with the number of snapshots. It is noted that the results from fig. 5 show that the more the snapshots the narrower is the beam width of the signal. Although the higher the number of snapshots the more accurate is the DOA estimation for the uniform linear array, the non-uniform linear array has better performance as shown in fig. 6 , fig. 7 and fig. 8.

The experiment 2 shows the variation of DOA with the number of array elements. For the smart antennas to obtain a reasonable gain, an array antenna with many elements is necessary. As observed in fig 4.9 the beam width becomes narrower as the number of array elements keeps on increasing. But the performance is better when using non-uniform linear array as observed in fig. 10, fig. 11 and fig. 12. Although a very big number of sensors lead to much computational complexity which demands more time for processing, also it may lead to a mutual coupling and may occupy a big space which makes it to be more expensive. Therefore, choosing a reasonable number of array elements is necessary to avoid problems stated above.

\section{CONCLUSION}

The direction of arrival (DOA) plays a very big important role in wireless communication systems particularly in array signal processing. It has many application in engineering fields such as radar, sonar, weather forecasting, tracking targets, ocean and geological exploration, seismic survey and biomedical, and communications in general.

From the simulation results, it is clear that the MUSIC algorithm is more accurate and has a higher resolution. This occurs especially when there is large size of antenna array, more SNR and a high number of snapshots for the ULA. In addition, when comparing the uniform linear array to the non-uniform linear array, the latter shows a higher accuracy and higher resolution. The NLA has more degree of freedom and allow a larger inter-element spacing than a half wavelength $\left(d>\frac{\pi}{2}\right)$ which is not the case for ULA. In addition, the autocorrelation of signals can be estimated in a much denser spacing other than the physically sparse sampling spacing, and sinusoids in noise can be estimated in a more effective way when using non-uniform linear array. The Non-uniform linear array is thus more advantageous than a uniform linear array.

Finally, the answer to some accuracy and directivity problems of direction of arrival estimation by using the MUSIC algorithm was given. The simulation experiments are given to perform some of those problems.

Briefly, this paper described what DOA estimation is, and give a mathematical model of DOA estimation. Then, the analysis of the performance for uniform linear array (ULA) and non-uniform linear array (NLA) were given. The estimation DOA based on the multiple classification signal (MUSIC) algorithm was provided. An extensive simulation has been conducted in MATLAB and the results show that the NLA for co-prime array can achieve an accurate and efficient DOA estimation.

In the future, the application of the two dimensions of NLA in form of DOA estimation for co-prime array is recommended in more accordance with the real time environments.

\section{REFERENCES}

[1]. Z. Tan, "Direction of arrival estimatiom using co-prime arrays: Asuper resolution viewpoint," IEEE transaction on signal processing , vol.62, no.21, pp. 5565 5576, November 2014.

[2]. R. Muhamed, Direction of arrival estimation using antenna arrays, Masters Thesis, Virginia Polytechnic Institute and State University, Electrical Engineering department, Blacksburg, Virginia, USA, January 1996. 
[3]. P. T. Eswar and K. Y. Krishna, "Performance analysis of direction of arrival estimation algorithms in smart antennas," International journal of advanced research in electronics and communication engineering, vol. III, no. 6, pp. 664 - 670, June 2014.

[4]. A. B. Nauman and B. M. Mohammad, "Comparison of direction of arrival (DOA) estimation techniques for closely spaced targets," International journal of future computer and communication, vol. II, no. 6, pp. 654-659, December 2013.

[5]. M. H. Mohammad, "Direction of arrival estimation using maximum likelihood method and the effect of element selection on system performance," Masters thesis, Concordia University, Electrical and Computer Engineering, Montreal, Quebec, Canada, August 2015.

[6]. R. K. Aniket, D.Ganage and S. Wagh, "Subspace based DOA estimation techniques," International journal of research in engineering and technology, vol. IV, no. 6, pp. 560 - 564, June 2015.

[7]. D. Yimin, Q. Si and G. Moeness, "DOA estimation exploiting co-prime arrays with sparse sensor spacing," Center for advanced communication, Villanova university, Pennyslvania, USA, 2015.

[8]. Z. Chenguei, Z. Shi, G. Yujie and S. Xuemin, "DECOM: DOA estimation for co-prime array," Wireless communications and signal processing (WCSP), 2013 International Conference, Yangzhou, Jiangsu, China, vol. v, no. 13, pp. 978 - 982, October 2013 IEEE.

[9]. P. Pal and P.Vaidyanathan, "A novel array structure for direction of arrival estimation with increased degrees of freedom," Accoustics speech and signal processing (ICASSP), 2010 International Conference, Dallas, Texas, USA, pp. 2606-2609, March 2010 IEEE.

[10]. P. Pal and P. Vaidyanathan, "Coprime sampling and the MUSIC algorithm," Digital signal processing workshop and IEEE signal processing education workshop (DSP/SPE), 2011 IEEE, Sedona, Arizona, USA, pp. 289-294, January 2011 IEEE.

[11]. W. Zhiyuan and M. D. Petar, "A searchfree DOA estimation algorithm for coprime arrays," Digital signal processing, Elsevier, vol. III, pp. 1 - 22, October 2014.

[12]. N. A. Dhering and B.Bansode, "Performance evaluation and analysis of direction of arrival estimation using MUSIC,TLS ESPRIT, and Pro ESPRIT algorithms," International journal of advanced research in electrical, electronics and instrumentation engineering, vol. IV, no. 6, pp. 4948 - 4958, June 2015. 